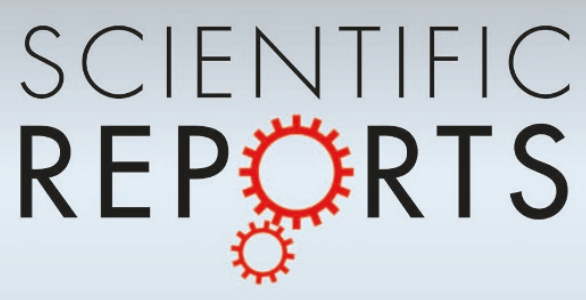
Negative Differential Resistance Effects

SUBJECT AREAS:

ELECTRONIC DEVICES

MOLECULAR ELECTRONICS

ELECTRONIC AND SPINTRONIC DEVICES

Received

22 August 2013

Accepted

31 March 2014

Published

2 May 2014

Correspondence and requests for materials should be addressed to

K.L.Y. (klyao@hust. edu.cn) \title{
in a $\mathrm{Fe}_{4}$-based Single-molecule Junction
}

Fengxia Zu' ${ }^{1}$, Zuli Liu', Kailun Yao',3, Guoying Gao' , Huahua Fu' , Sicong Zhu' , Yun Ni' \& Li Peng'

'School of Physics and Wuhan National High Magnetic Field Center, Huazhong University of Science and Technology, Wuhan 430074, China, ${ }^{2}$ School of Science, Wuhan Institute of Technology, Wuhan 430073, China, ${ }^{3}$ International Center of Materials Physics, Chinese Academy of Science, Shenyang 110015, China.

The spin-polarized transport in a single-molecule magnet $\mathrm{Fe}_{4}$ sandwiched between two gold electrodes is studied, using nonequilibrium Green's functions in combination with the density-functional theory. We predict that the device possesses spin filter effect (SFE), spin valve effect (SVE), and negative differential resistance (NDR) behavior. Moreover, we also find that the appropriate chemical ligand, coupling the single molecule to leads, is a key factor for manipulating spin-dependent transport. The device containing the methyl ligand behaves as a nearly perfect spin filter with efficiency approaching $100 \%$, and the transport is dominated by transmission through the $\mathrm{Fe}_{4}$ metal center. However, in the case of phenyl ligand, the spin filter effect seems to be reduced, but the spin valve effect is significantly enhanced with a large magnetoresistance ratio, reaching $1800 \%$. This may be attributed to the blocking effect of the phenyl ligands in mediating transport. Our findings suggest that such a multifunctional molecular device, possessing SVE, NDR and high SFE simultaneously, would be an excellent candidate for spintronics of molecular devices.

M olecular spintronics using molecules as spin transport elements has attracted intensive interest due to obtained various functionality, and molecular devices such as spin valve, single-molecule transistors, switch and diode, etc $^{1-5}$. Conventionally, the magnetic molecule junction (MMJ) can be achieved by placing a nonmagnetic molecular bridge between two ferromagnetic electrodes ${ }^{6,7}$. In the rather newer field, MMJ can also be achieved by coupling a magnetic molecule to two nonmagnetic probes ${ }^{8-10}$. In this approach, singlemolecule magnets (SMMs) are ideal building blocks to construct MMJ because of their independent magnetically functional units ${ }^{11-13}$, where the inner magnetic core in SMMs is surrounded by organic ligands and the interaction between magnetic cores of neighbor molecule is very weak, leading to a large-spin ground state. Unlike above mentioned conventional MMJ depending on reasonable magnetic configuration of two ferromagnetic leads $\mathrm{d}^{7,14}$, the origin of magnetic behavior in this MMJ consisting of a single-molecule magnet and two nonmagnetic electrodes is the intrinsic spin of the center magnetic molecule, therefore avoiding the great difficulty of the spin injection from ferromagnetic electrodes into a nonmagnetic center in real applications. And so the unique feature of MMJ containing SMM would offer much greater potential for high-density information storage devices ${ }^{15}$.

Although there have been some successful efforts to organize SMMs on various substrates, the observation of magnetic hysteresis on individual molecules organized on surfaces ${ }^{16,17}$, that is a necessary step to develop molecular memory arrays, had not been reported for monolayers of SMMs when wired to metallic surfaces until Matteo Mannini and co-workers found that tailor-made tetranuclear $\mathrm{Fe}_{4}$ complexes retain their magnetic properties at gold surfaces ${ }^{15}$. This encouraging result naturally excites our attention to investigate quantum transport through this high-performance magnetic molecule $\mathrm{Fe}_{4}$. Several recent works mainly focused on the charge transport and the magnetic anisotropy in a single-electron transistor containing this magnetic molecule ${ }^{18-20}$. However, to the best of our knowledge, the spin-polarized transport on this magnetic molecule has not been reported both theoretically and experimentally so far. Here, we carry out first-principles transport calculations through the single magnetic molecule $\mathrm{Fe}_{4}$ linked to two gold nanowires. Furthermore, we firstly explore the potential of a spinpolarized current through this magnetic molecule to read and write spin-based information. We predict that this device has a nearly perfect spin filter effect with efficiency approaching $100 \%$. Moreover, it also shows spin valve behavior and negative differential resistance effect. In addition, we also find the ligand group coupling the molecule to leads is a key factor for manipulating spin dependent transport. These findings strongly suggest that such a multifunctional molecular device would be candidate for molecular spintronics. 


\section{Results}

The structure of $\mathrm{Fe}_{4}$ molecule is shown in Fig. 1(a), where the metallic cores are composed of a triangle of iron ions and an inner ion ${ }^{21}$. Two derivatives are studied: $\mathrm{Fe}_{4} \mathrm{Me}$ functionalized with tripodal ligands methyl- $\mathrm{C}\left(\mathrm{CH}_{2} \mathrm{OH}\right)_{3}$ (See Fig. 1(b)), and $\mathrm{Fe}_{4} \mathrm{Ph}$ functionalized with phenyl- $\mathrm{C}\left(\mathrm{CH}_{2} \mathrm{OH}\right)_{3}$ (See Fig. 1(c)). The two ligands have been shown to be advantageous for deposition of $\mathrm{Fe}_{4}$ molecule on gold surfaces $^{18,19}$. The device we considered consists of two semi-infinite gold electrodes sandwiching each $\mathrm{Fe}_{4}$ derivative via thiol end groups, as shown in Fig. 1(b) and (c). The gold electrodes extend to $z= \pm \infty$ along the transport direction, and extend with large vacuum layer in transverse $x$ and $y$ directions in order to suppress the interaction between transport system and its mirror images.

First, we study the transport properties of $\mathrm{Fe}_{4} \mathrm{Me}$ derivative, where the $\mathrm{Fe}_{4}$ core is connected to the gold nanoelectrodes via methyl ligands terminated with thiol groups as shown in Fig. 1(b). Two magnetic structures are considered for the spin-dependent transport properties. One is antiparallel configuration (APC) as depicted in the left panel of Fig. 1(a), where the outer individual spins interact antiferromagnetically with the inner one, to give a $S=5$ ground spin state. Another is parallel configuration (PC) as seen in the right panel of Fig. 1(a), where all the four iron cores have the parallel spin direction, to give a $S=10$ high-spin state. A magnetic field can switch the $\mathrm{Fe}_{4}$ molecule from one state to the other, and the further calculations indicates that the magnitude of magnetic field needed to switch the molecule from the antiferromagnetic state to the ferromagnetic state is about $32 \mathrm{~T}$, which is comparable with the results obtained by Baranger et $a l^{8}$. Fig. 2(a) and 2(b) show the current-voltage (I-V) curves of the magnetic molecular junction for APC and PC, respectively. The total current for APC and $\mathrm{PC}$ are rather different, resulting in a large magnetoresistance $(\mathrm{MR})$ ratio defined as $\mathrm{MR}=\left(\mathrm{I}_{\mathrm{APC}}-\right.$ $\left.I_{P C}\right) / I_{P C}$ as shown in Fig. 2(c), where $I_{A P C}$ and $I_{P C}$ are the total currents for APC and PC related to the same applied voltage, respectively. At zero bias when all currents vanish, $\mathrm{MR} \sim 75 \%$ is obtained by using equilibrium conductance. This behavior shows that the device

(a)
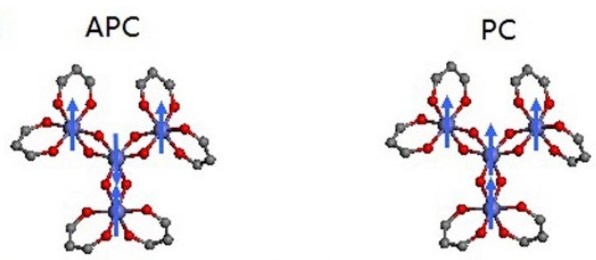

(b)

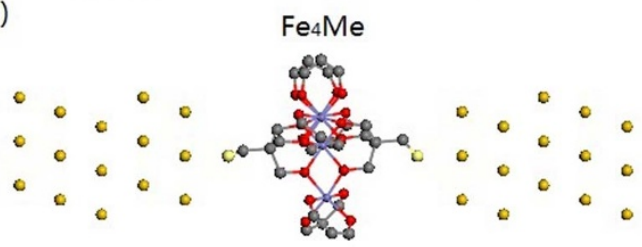

(c)

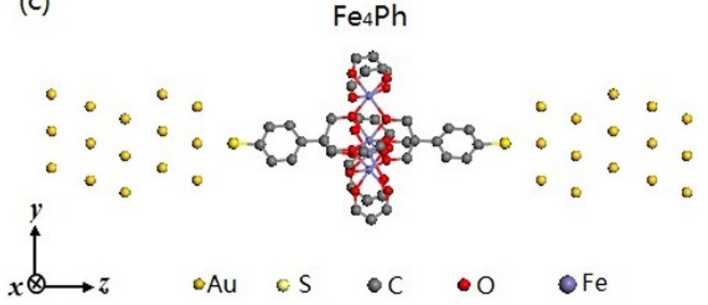

Figure 1 The schematic illustration of the SMM device. (a) A view of the magnetic core structure of $\mathrm{Fe} 4$ with antiparallel configuration (APC) and parallel configuration (PC). (b) $\mathrm{Fe}_{4} \mathrm{Me}$ functionalized with methyl ligands, and (c) $\mathrm{Fe}_{4} \mathrm{Ph}$ functionalized with phenyl ligand are respectively bonded to the two semi-infinite Au leads via thiol end groups. H atom is not shown for the sake of clarity. can also be used as an organic spin valve. The calculated zero-bias conductance for APC remains essentially the same magnitude as the experimental results ${ }^{18}$. Furthermore, Fig. 2(b) displays that the total currents for PC are only from the spin-down channel, the spin-up current is completely suppressed, leading to a good spin filter effect. Also, a spin-filter diagram (see Fig. 1 in Ref. 22) can be used to describe how the spin current in Fig. 2 (b) tunnels through the device. The spin filter efficiency defined as $\eta=\left(I_{\downarrow}-I_{\uparrow}\right) /\left(I_{\downarrow}+I_{\uparrow}\right)$ for PC is higher than $99 \%$ in the whole bias region as shown in Fig. 2(d), indicating a perfect SFE. In addition, as seen from Fig. 2(a) and 2(b), the total currents for APC and PC increase nonlinearly with increasing bias voltage, and reach a maximum around $5.6 \mathrm{mV}$. Afterward, the total currents decline when the applied bias voltage is increased to larger values, leading to a negative differential resistance (NDR) behavior.

It is well known that the ligand coupling the molecule to the leads is a crucial issue for the preparation of single-molecule electronic device. Here, we also investigate the transport properties of $\mathrm{Fe}_{4} \mathrm{Ph}$ derivative by substituting the methyl groups in $\mathrm{Fe}_{4} \mathrm{Me}$ with phenyl ligands (See Fig. 1(c)). The calculated results for spin parallel configuration and antiparallel configuration are given in Fig. 3. Compared with the above-mentioned $\mathrm{Fe}_{4} \mathrm{Me}$ in Fig. 2, the transport property of $\mathrm{Fe}_{4} \mathrm{Ph}$ derivative shows two main differences: (1) The strong spin polarization appears in APC rather than in PC, different from the situation in $\mathrm{Fe}_{4} \mathrm{Me}$ depicted in Fig. 2(a) and 2(b). Furthermore, we discover that the spin filter efficiency for PC in $\mathrm{Fe}_{4} \mathrm{Me}$ (See Fig. 2(d)), is higher than that for APC in $\mathrm{Fe}_{4} \mathrm{Ph}$ (See Fig. 3(d)), which indicates the methyl groups bridging the $\mathrm{Fe}_{4}$ molecule and electrodes are more advantageous for the spin filter effect than the phenyl ligands. (2) In $\mathrm{PC}$ of $\mathrm{Fe}_{4} \mathrm{Ph}$ system, both the spin-up and the spin-down currents are limited, and thus the total current for APC is much larger than that for PC, exhibiting a substantial magnetoresistance with a maximum $1800 \%$ (See Fig. 3(c)), which is significantly larger than that observed in $\mathrm{Fe}_{4} \mathrm{Me}$. This suggests that the phenyl ligand is highly advantageous for the spin valve effect.

\section{Discussion}

To further explore the underlying mechanisms of MR and SFE in $\mathrm{Fe}_{4} \mathrm{Me}$ device, Fig. 4(a) and 4(b) present the spin-resolved transmission coefficient $\mathrm{T}(E)$ and the spatial distribution of the molecular projected self-consistent Hamiltonian states (MPSHs) of the HOMO (highest occupied molecular orbital) and LUMO (lowest unoccupied molecular orbital) at zero bias. For APC, the T(E) of Fig. 4(a) shows that the total current comes from both the spin-up and the spindown transmission peaks around the Fermi level. Furthermore, MPSHs in Fig. 4(a) reveal that the outer three iron ions provide the spin-down transmission channels, and the centered iron ion contributes to the spin-up channel. Whereas, for PC in Fig. 4(b), $\mathrm{T}(E)$ and MPSHs indicate that the resonant transmission peaks are only from the spin-down electrons of the four iron ions, the spin-up $\mathrm{T}(E)$ is essentially zero around the Fermi energy, which suggests that only spins parallel to the molecular magnetization can flow through the SMM, exhibiting a very high spin polarization with efficiency approaching $100 \%$. Simultaneously, this also results in that the total current in APC is significantly larger than that in PC. And so the device can be used for a spin valve by switching between APC and PC magnetization configurations under an applied magnetic field.

In order to explore the physical origin of the NDR phenomenon, we investigate the spin-resolved transmission properties around the Fermi level. Since the NDR behaviors are found for APC and PC almost at the same bias region, we only give the transmission coefficient of PC system at $6.8 \mathrm{mV}$ and $5.6 \mathrm{mV}$, as shown in Fig. 5(a) and 5 (b), respectively. One can see that the main transmission peak, corresponding to the conduction orbital LUMO (lowest unoccupied molecular orbital) above the Fermi energy, is responsible for the NDR behavior. The LUMO peak originating mainly from the $\mathrm{d}_{\mathrm{z}}{ }^{2}$ 

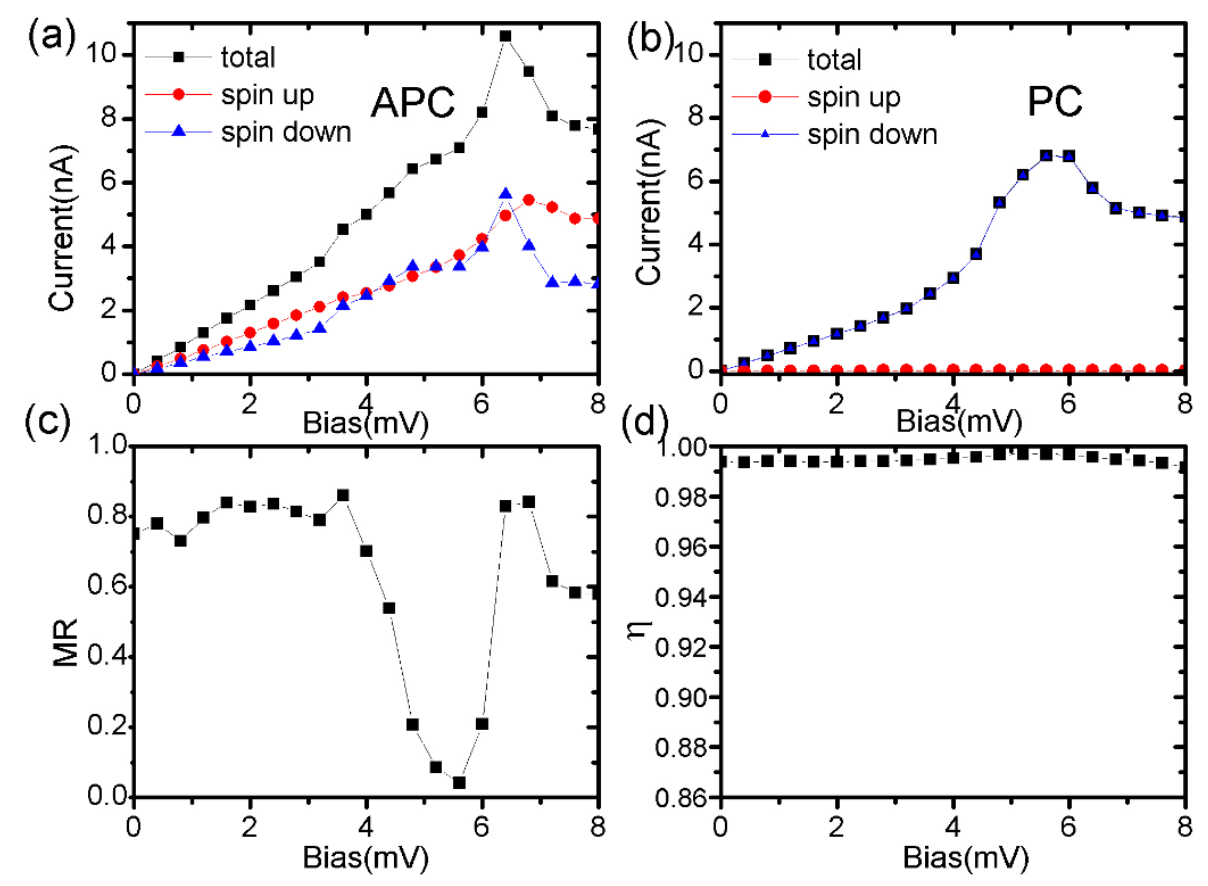

Figure $2 \mid$ The transport properties of Fe4Me. (a) and (b) Current-voltage characteristics for magnetic antiparallel configuration and parallel configuration, respectively.Total means the sum of the spin-up and spin-down currents for each magnetic configuration. (c) Magnetoresistance ratio. (d) Spin filter efficiency for parallel configuration.

orbitals of four Fe cores is higher in Fig. 5(b) than in Fig. 5(a). In addition, we notice that a short transmission peak appears at $0.08 \mathrm{eV}$ in Fig. 5(b), which arises from the hybrid state between the molecule and the electrode surface, whereas, the peak is not found in Fig. 5(a). Therefore, the hybrid state is another reason for the NDR behavior.

Now, let us turn attention to the influence of the ligand on the transport properties. The local density of states (LDOS), as depicted in Fig. 6, provides valuable information, which can be helpful in deciding which parts of the chemical structure should be responsible for the transmission. First, we study the local contributions to transmission for the $\mathrm{Fe}_{4} \mathrm{Me}$ system. For APC, This is, in fact, exactly what is seen in Fig. 6(a) and 6(b), the spin down density is localized on the outer three iron ions, and likewise, the spin up electron density is localized on the centered iron ion with only a little distribution on the outer three iron ions. The fact indicates that the total transmission in APC is from the sum of contributions of the spin-up and spin-down
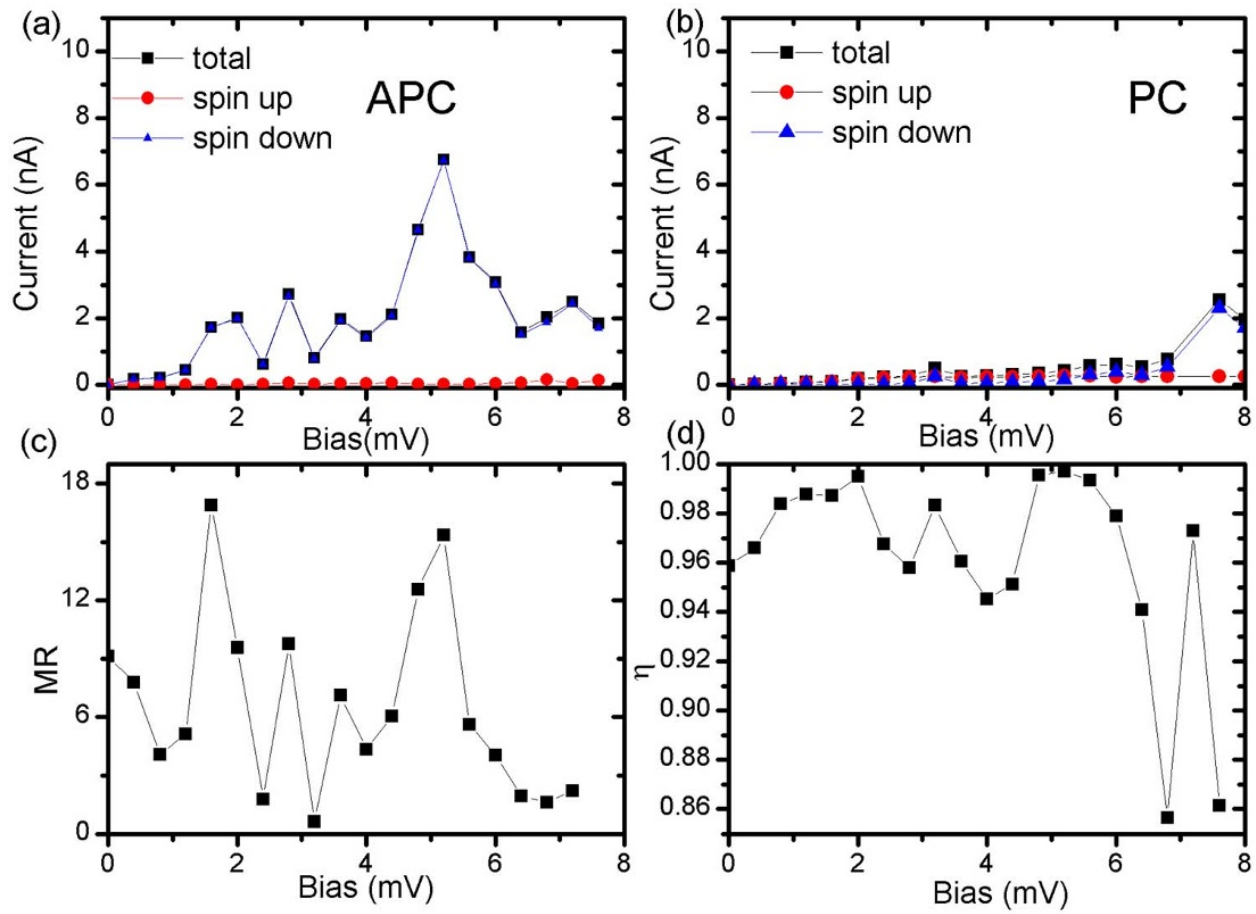

Figure $3 \mid$ The transport properties of Fe4Ph. (a) and (b) Current-voltage characteristics for magnetic antiparallel configuration and parallel configuration, respectively.(c) Magnetoresistance ratio. (d) Spin filter efficiency for parallel configuration. 

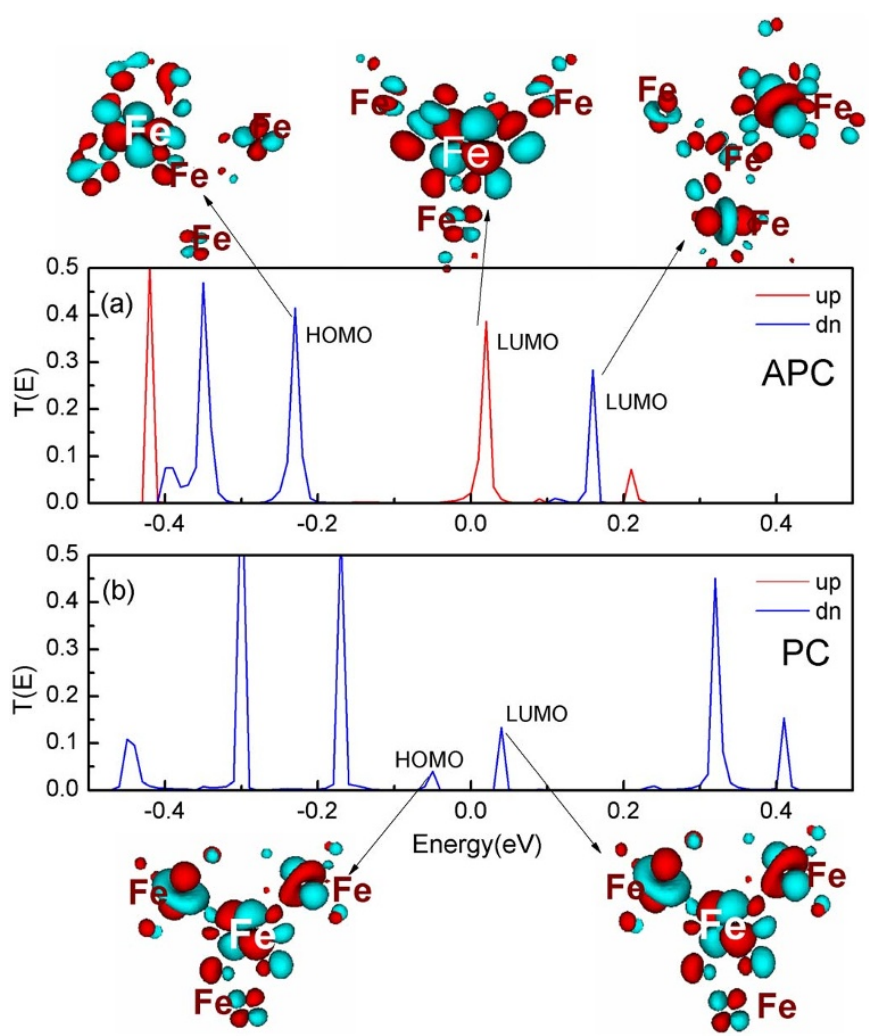

Figure 4 Spin-polarized transmission coefficient and MPSH states of $\mathrm{Fe}_{4} \mathrm{Me}$ at zero bias (a) for antiparallel configuration and (b) for parallel configuration. The average Fermi level is set to zero.

electrons. For PC, Fig. 6(c) and 6(d) illustrate that only one spin direction (i.e. spin up) of the conducting electrons is transmitted, leading to a nearly perfect spin filter effect, simultaneously, explaining the occurrence of spin-valve behavior. These behaviors are in line with the results from the energy spectrum of the molecular projected self-consistent Hamiltonian (MPSH) at zero bias (Supporting Information, Figure S1). In general, the transport in $\mathrm{Fe}_{4} \mathrm{Me}$ system whether for APC or PC is dominated by transmission through the $\mathrm{Fe}_{4}$ metal center, less sensitive to the methyl ligand. However, the case is different in $\mathrm{Fe}_{4} \mathrm{Ph}$ device, except for Fig. 6(e), where the metal centers are still the main contributor for the transmission. From Fig. 6(f) to 6(h), and in particular for Fig. 6(f), the through-metal contribution is well below the through-ligand contributions. Furthermore, for all

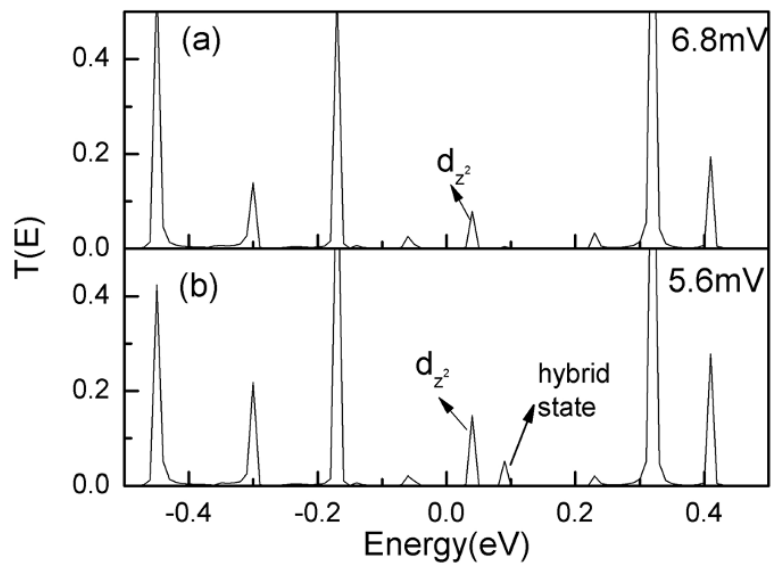

Figure $5 \mid$ The bias-dependent transmission coefficient of $\mathrm{Fe}_{4} \mathrm{Me}$ in parallel configuration (a) for $6.8 \mathrm{mV}$ and (b) for $5.6 \mathrm{mV}$. The spin-up transmission coefficient is almost zero.
Spin-down

Spin-up

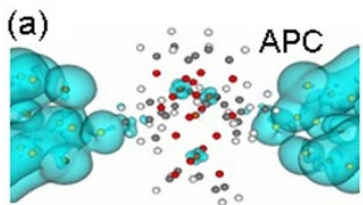

(c)
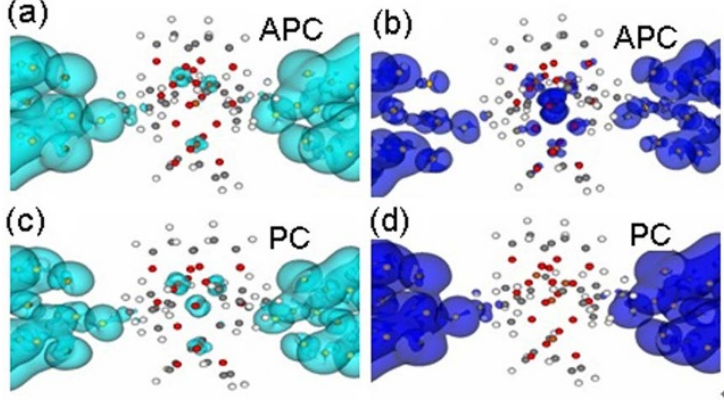

(d)

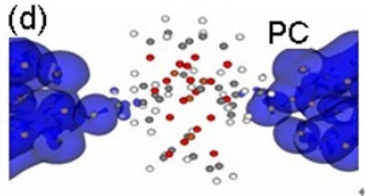

(e)
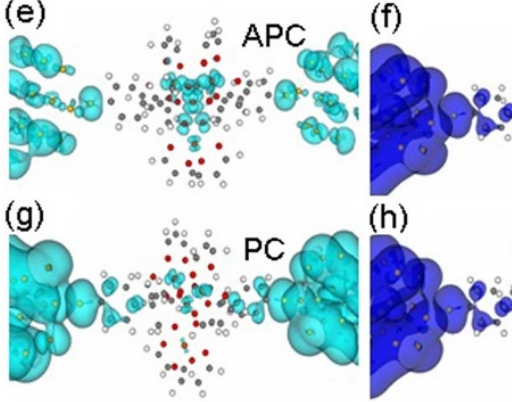

(h)

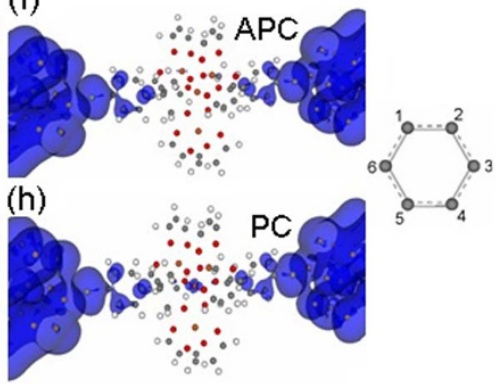

Figure $6 \mid$ Surfaces of constant LDOS at the Fermi energy. $(a / b)$ and $(c / d)$ for device $\mathrm{Fe}_{4} \mathrm{Me}$ depict density of states in antiparallel configuration and parallel configuration, respectively. $(\mathrm{e} / \mathrm{f})$ and $(\mathrm{g} / \mathrm{h})$ are similar to $(\mathrm{a} / \mathrm{b})$ and (c/d), but for $\mathrm{Fe}_{4} \mathrm{Ph}$. The inset on the right side of (f) shows the sites of carbon atoms in phenyl ring. Green (blue) represents spin down (spin up) density of states.

three cases, it is found that the densities are localized on 1, 3, 5 sites in the left phenyl ring, forming the conjugated $\pi$ bonding orbital, and likewise, the densities are localized on 2, 4, 6 sites in the right phenyl ring, forming the $\pi^{*}$ antibonding orbital. Since the antibonding orbital has higher energy than the bonding orbital, it is difficult that either spin from the left phenyl ring penetrates into the right one, and hence, the conductance is low. This implies that the blocking effect of the phenyl ligands on the electronic transport in Fig. 6(f) to 6(h), is mainly responsible for the large spin valve and spin filer effects in $\mathrm{Fe}_{4} \mathrm{Ph}$ system.

\section{Methods}

The spin-dependent electron transport calculations are performed using a recently developed package Atomistix Toolkit ${ }^{23-25}$, which combines the nonequilibrium Green's-function (NEGF) with a self-consistent density-functional theory (DFT) ${ }^{26,27}$ The generalized gradient approximation (GGA) in Perdew-Burke-Ernzerhof (PBE) format ${ }^{28}$ is adopted for the exchange correlation potential in DFT. Further details of the method were presented in Ref. 29. The wave functions are expanded on a numerical basis set of double zeta-polarized basis. The mesh cut-off energy is $150 \mathrm{Ry}$ and the convergent results are achieved by using the k-points sampling 100 in transport $\mathrm{z}$ direction. This procedure is iterated until it reaches self-consistency when the convergence criterion $10^{-5}$ for the total energy is achieved to present an accurate charge density. The geometry of the scattering region is relaxed when the force tolerance of $0.05 \mathrm{eV} / \AA$ is achieved, where the outmost layers of $\mathrm{Au}$ atoms are fixed at their bulk positions. After the self-consistent NEGF-DFT procedure is converged, we obtain spin-polarized current using the Landauer-Buttiker formula:

$$
I_{\sigma}=\frac{e}{h} \int_{-\infty}^{+\infty} T_{\sigma}\left(E, V_{b}\right)\left[f_{L}\left(E-\mu_{L}\right)-f_{R}\left(E-\mu_{R}\right)\right] d E,
$$

where $f_{L, R}$ is the Fermi-Dirac distribution and $\mu_{L, R}$ are the chemical potentials of the left electrode $(L)$ and the right electrode $(R)$, respectively. $T_{\sigma}\left(E, V_{b}\right)$ is the spindependent transmission coefficient at the energy $E$ with bias voltage $V_{b}$.

Finally, we attempt to check the DFT results by using a different exchange correlation functional. For comparison, we also apply the local density approximation (LDA) in Perdew-Zunger parametrization as the exchange correlation functional, and investigate the transport properties of $\mathrm{Fe}_{4} \mathrm{Me}$ device as an example. The calculated I-V curves with the LDA functional are provided in the Supporting Information 
(Figure S2), where one can observe the NDR peak whether for APC or PC are shifted to higher energies (compare the NDR peaks with the GGA functional in Fig. 2(a) and Fig. 2(b)). Simultaneously, one can see that the current value (a maximum of $18 \mathrm{nA}$ ) with the GGA functional is larger than that (a maximum of $12 \mathrm{nA}$ ) with the LDA. Accordingly, a MR maximum of $\sim 80 \%$ with GGA is reduced to $\sim 60 \%$ with LDA. However, the overall shape of these transmission curves using LDA remains essentially the same as those using GGA. Furthermore, a MR maximum of $\sim 85 \%$ obtained with LDA is also in line with results from GGA, though the calculated current with LDA is larger than that with GGA. This indicates that changing the functional will result in quantitative changes in the transmission, but not affect our qualitative conclusions, such as the nature of the NDR, spin valve, and spin filter effects.

1. Nguyen, T. D., Ehrenfreund, E. \& Vardeny, Z. V. Spin-Polarized Light-Emitting Diode Based on an Organic Bipolar Spin Valve. Science 337, 204-209 (2012).

2. Zhu, L., Yao, K. L. \& Liu, Z. L. Magnetic and electronic switching properties of photochromic diarylethene with two nitronyl nitroxides. Appl. Phys. Lett. 97, 202101 (2010)

3. Tu, X. W., Mikaelian, G. \& Ho, W. Controlling Single-Molecule Negative Differential Resistance in a Double-Barrier Tunnel Junction. Phys. Rev. Lett. 100, 126807 (2008).

4. Ni, Y. et al. Spin Seebeck Effect and Thermal Colossal Magnetoresistance in Graphene Nanoribbon Heterojunction. Sci. Rep. 3, 1380 (2013)

5. Chae, D. H. et al. Vibrational Excitations in Single Trimetal-Molecule Transistors. Nano Lett. 6, 165-168 (2006).

6. Rocha, A. R. et al. Towards molecular spintronics. Nat. Mater. 4, 335-339 (2005).

7. Ning, Z. Y., Zhu, Y., Wang, J. \& Guo, H. Quantitative Analysis of Nonequilibrium Spin Injection into Molecular Tunnel Junctions. Phys. Rev. Lett. 100, 056803 (2008).

8. Liu, R., Ke, S. H., Baranger, H. U. \& Yang, W. T. Organometallic Spintronics: Dicobaltocene Switch. Nano Lett. 5, 1959-1962 (2005).

9. Urdampilleta, M., Klyatskaya, S., Cleuziou, J.-P., Ruben, M. \& Wernsdorfer, W. Supramolecular spin valves. Nat. Mater. 10, 502-506 (2011).

10. Georgiev, V. P. \& McGrady, J. E. Efficient Spin Filtering through Cobalt-Based Extended Metal Atom Chains. Inorg. Chem. 49, 5591-5597 (2010).

11. Heersche, H. B., de Groot, Z., Folk, J. A. \& van der Zant, H. S. J. Electron Transport through Single $\mathrm{Mn}_{12}$ Molecular Magnets. Phys. Rev. Lett. 96, 206801 (2006)

12. Vincent, R., Klyatskaya, S., Ruben, M., Wernsdorfer, W. \& Balestro, F. Electronic read-out of a single nuclear spin using a molecular spin transistor. Nature 488, 357-360 (2012).

13. Jo, M. H. et al. Signatures of Molecular Magnetism in Single-Molecule Transport Spectroscopy. Nano Lett. 6, 2014-2020 (2006).

14. Horiguchi, K., Sagisaka, T., Kurokawa, S. \& Sakai, A. Electron transport through $\mathrm{Ni} / 1,4$-benzenedithiol/Ni single-molecule junctions under magnetic field. J. Appl. Phys. 113, 144313 (2013).

15. Mannini, M. et al. Magnetic memory of a single-molecule quantum magnet wired to a gold surface. Nat. Mater. 8, 194-197 (2009).

16. Mannini, M. et al. XAS and XMCD Investigation of $\mathrm{Mn}_{12}$ Monolayers on Gold. Chem. Eur. J. 14, 7530-7535 (2008)

17. Mannini, M. et al. X-Ray Magnetic Circular Dichroism Picks out Single-Molecule Magnets Suitable for Nanodevices. Adv. Mater. 21, 167-171 (2009).

18. Zyazina, A. S., van der Zanta, H. S. J., Wegewijsb, M. R. \& Corniae, A. High-spin and magnetic anisotropy signatures in three-terminal transport through a single molecule. Synth.Met. 161, 591-597 (2011).

19. Zyazin, A. S. et al. Electric Field Controlled Magnetic Anisotropy in a Single Molecule. Nano Lett. 10, 3307-3311 (2010).
20. Burzurı, E., Zyazin, A. S., Cornia, A. \& van der Zant, H. S. J. Direct Observation of Magnetic Anisotropy in an Individual $\mathrm{Fe}_{4}$ Single-Molecule Magnet. Phys. Rev. Lett. 109, 147203 (2012).

21. Accorsi, S. et al. Tuning Anisotropy Barriers in a Family of Tetrairon(III) SingleMolecule Magnets with an S = 5 Ground State. J. Am. Chem. Soc. 128, 4742-4755 (2006).

22. José María De Teresa. Double or nothing for spin filters. Physics 2, 13 (2009).

23. Taylor, J., Guo, H. \& Wang, J. Ab initio modeling of quantum transport properties of molecular electronic devices. Phys. Rev. B 63, 245407 (2001).

24. Brandbyge, M., Mozos, J. L., Ordejón, P., Taylor, J. \& Stokbro, K Densityfunctional method for nonequilibrium electron transport. Phys. Rev. B, 65, 165401 (2002).

25. Soler, J. M. et al. The SIESTA method for ab initio order-N materials simulation. J. Phys. Condens. Matter. 14, 2745 (2002).

26. Fu, H. H. \& Yao, K. L. Perfect spin-filter and highly spin-polarized current in a quantum network device. Appl. Phys. Lett. 100, 013502 (2012).

27. Fu, H. H. \& Yao, K. L. Spin-polarized transport through a parallel triple-quantumdot device: Blockade effects of Rashba spin-orbit interaction and Coulomb interaction. J. Appl. Phys. 110, 094502 (2011).

28. Perdew, J. P., Burke, K. \& Ernzerhof, M. Generalized Gradient Approximation Made Simple. Phys. ReV. Lett. 3865, 77 (1996).

29. Ke, S. H., Baranger, H. U. \& Yang, W. Self-consistent and Electron transport through molecules: non-self-consistent approaches. Phys. Rev. B. 70, 085410 (2004).

\section{Acknowledgments}

The authors would like to acknowledge the support from the National Natural Science Foundation of China (Grant No. 11074081, No. 11004066, No. 11274130, No. 11274127 and 11274128).

\section{Author contributions}

F.Z. designed the magnetic molecule junction and wrote the manuscript. K.Y. put forward the idea and supervised the whole work. F.Z., G.Y., S.Z. and Y.N. carried out the numerical calculations. F.Z., G.Y. and H.F. analyzed the results. Z.L. and L.P. made a discussion. All authors reviewed the manuscript.

\section{Additional information}

Supplementary information accompanies this paper at http://www.nature.com/ scientificreports

Competing financial interests: The authors declare no competing financial interests. How to cite this article: Zu, F.X. et al. Nearly Perfect Spin Filter, Spin Valve and Negative Differential Resistance Effects in a $\mathrm{Fe}_{4}$-based Single-molecule Junction. Sci. Rep. 4, 4838; DOI:10.1038/srep04838 (2014).

This work is licensed under a Creative Commons Attribution-NonCommercialNoDerivs 3.0 Unported License. The images in this article are included in the article's Creative Commons license, unless indicated otherwise in the image credit; if the image is not included under the Creative Commons license, users will need to obtain permission from the license holder in order to reproduce the image. To view a copy of this license, visit http://creativecommons.org/licenses/by-nc-nd/3.0/ 